Recherches amérindiennes au Québec

\title{
Witamowikok, « dire » le territoire atikamekw nehirowisiw aujourd'hui
}

Territoires de l'oralité et nouveaux médias autochtones

Witamowikok, “Telling” Atikamekw Nehirowisiw Land Today

Territories of Orality and Aboriginal New Media

Witamowikok, " decir » el territorio atikamekw nehirowisiw hoy

Territorios de la oralidad y nuevos medios de comunicación indígenas

Witamakewin anotc e icinakok atikamekw nehirowisiw otaskik

Askik e ici wawitamakaniwok kekwan e motci aiarimwaniwok nac nihe

e iti apatak kaskina anotc ka apatcitakaniwok irapatcitcikan e tipatcimowakaniwok kekwan

Laurent Jérôme et Vicky Veilleux

Volume 44, numéro 1, 2014

Les Atikamekw Nehirowisiwok : territorialités et savoirs

URI : https://id.erudit.org/iderudit/1027876ar

DOI : https://doi.org/10.7202/1027876ar

Aller au sommaire du numéro

Éditeur(s)

Recherches amérindiennes au Québec

ISSN

0318-4137 (imprimé)

1923-5151 (numérique)

Découvrir la revue

Citer cet article

Jérôme, L. \& Veilleux, V. (2014). Witamowikok, « dire » le territoire atikamekw nehirowisiw aujourd'hui : territoires de l'oralité et nouveaux médias autochtones. Recherches amérindiennes au Québec, 44(1), 11-22. https://doi.org/10.7202/1027876ar

\section{Résumé de l'article}

Depuis trente ans, les Nehirowisiwok sont engagés dans un processus de revendication territoriale avec les gouvernements québécois et canadien. Cet article vise à documenter l'une des voies privilégiées de leur démarche de résistance, d'autodétermination et de reconnaissance de la souveraineté : celle du discours. La prise de parole est considérée comme un acte créatif à l'intérieur duquel s'exprime toute la capacité d'agir des Nehirowisiwok. Des récits récoltés lors de recherches de terrain réalisées depuis 2000 en territoire atikamekw nehirowisiw, ainsi que la production audiovisuelle atikamekw nehirowisiw du projet Wapikoni Mobile, constituent différentes sources permettant de relever les conceptions et les préoccupations des Nehirowisiwok d'aujourd'hui à l'égard de leur territoire. Avant de s’intéresser aux modalités d'appropriation des nouveaux médias par les autochtones, en particulier par les jeunes Nehirowisiwok, les auteurs ancrent d'abord leur propos dans des éléments de continuité de la tradition orale, pour ensuite valoriser le « dire " que des aînés témoins des transformations du territoire adressent aux nouvelles générations de Nehirowisiwok et aux non-autochtones.
Tous droits réservés @ Recherches amérindiennes au Québec, 2014
Ce document est protégé par la loi sur le droit d'auteur. L’utilisation des services d’Érudit (y compris la reproduction) est assujettie à sa politique d'utilisation que vous pouvez consulter en ligne.

https://apropos.erudit.org/fr/usagers/politique-dutilisation/ 


\section{Laurent} Jérôme

Département de sciences des religions, Université du Québec à Montréal,

et

\section{Vicky Veilleux}

Assistante de recherche ${ }^{1}$
Witamowikok aka wiskat eki otci pakitinamokw kitaskino, nama wiskat ki otci atawanano, nama wiskat ki otci meckotonenano, nama kaie wiskat ki otci pitoc irakonenano Kitaskino.

Dites-leur que nous n'avons jamais cédé notre territoire, que nous ne l'avons jamais vendu, que nous ne l'avons jamais échangé, de même que nous n'avons jamais statué autrement en ce qui concerne notre territoire.

(César Newashish, 7 avril 1994)

ETTE DÉCLARATION a été prononcée par César Newashish le 7 avril 1994 depuis son lit d'hôpital, à Joliette, quelques mois avant sa mort (le 6 septembre 1994). César Newashish était alorsle doyen des Nehirowisiwok. Il avait 91 ans. Recueillie par Gilles Ottawa, historien nehirowisiw autodidacte, cette déclaration est devenue un cri de ralliement national autour de la protection et de la mise en valeur du Nitaskinan, le territoire ancestral atikamekw nehirowisiw. Gilles Ottawa était alors membre de l'équipe de négociation du Conseil de la Nation atikamekw (CNA). Il rendait visite à César Newashish pour l'informer que sa contribution n'était plus requise dans une cause portant sur les droits ancestraux. Les charges portées, en 1993, contre un Nehirowisiw de Manawan, accusé d'avoir trappé la martre en dehors des périodes autorisées par les lois et règlements sur la conservation de la faune du gouvernement du Québec, avaient finalement été abandonnées.
Né le 18 décembre 1902 sur le Nitaskinan, César Newashish avait quatre ans le 29 août 1906 lorsque la réserve de Manawan fut officiellement créée à la demande de son grand-père Louis Newashish et après des années de discussion avec le gouvernement fédéral. Régulièrement reprise dans les radios communautaires, affichée sur les murs des conseils de bande et dans les écoles atikamekw ou présentée en préambule de mémoires politiques déposés à l'Assemblée nationale du Québec, la déclaration de César Newashish illustre l'esprit de résistance des Nehirowisiwok. Depuis trente ans, on l'aura compris à la lecture de l'introduction et des articles de ce numéro, les Nehirowisiwok sont engagés dans un interminable processus de revendications avec les deux paliers de gouvernement. S'inscrivant dans le changement des politiques fédérales des années soixante-dix, époque où les politiques d'assimilation ont fait place aux politiques d'autodétermination, en 2014 les revendications territoriales atikamekw nehirowisiwok n'ont toujours pas abouti.

Le terme witamowikok, qui ouvre la déclaration de César Newashish, sert de pierre angulaire à cet article. Traduit en français par "dites-leur», witamowikok contient l'idée d'exprimer, de transmettre un message (Nicole Pétiquay, technolinguiste du Conseil de la Nation atikamekw, comm. pers., 7 mars 2014). Mais le locuteur 
délègue également une responsabilité à l'auditeur, soit celle de transmettre et de diffuser à son tour le message. Alors que les articles présentés dans ce numéro présentent différents aspects des relations que les Nehirowisiwok entretiennent avec le Nitaskinan, notre objectif est ici de livrer un regard sur la manière dont les Nehirowisiwok parlent du Nitaskinan aujourd'hui et relaient, à leur façon, le message de César Newashish. Nous proposons de documenter différentes formes et stratégies de prise de parole ainsi que de relever, dans ces discours, les conceptions et les préoccupations des Nehirowisiwok à l'égard de leur territoire. Qu'est-ce que les Nehirowisiwok disent de leur territoire aujourd'hui?

Sur le plan théorique, nous inscrivons cette analyse dans le débat sur les politiques de l'identité et de la culture en milieu autochtone en considérant les stratégies de prise de parole comme des actes créatifs de résistance et d'affirmation d'une souveraineté (sur ce sujet, voir Gagné et Salaün 2010). Pour de nombreux auteurs, la résistance a de nombreux points communs avec le pouvoir. Elle peut s'articuler autant dans la pratique que dans le discours (Bleiker 2000; Foucault 2005; Scott 1990). Nous suivons ici la perspective de Ortner pour laquelle

[...] on ne peut être en mesure de reconnaître les voies qui permettent à la résistance d'être plus que de l'opposition et d'être réellement créative et transformative qu'en reconnaissant la multiplicité des projets dans lesquels les humains sont constamment engagés, ainsi que la multiplicité des façons par lesquelles ces projets se nourrissent mutuellement et se heurtent les uns aux autres (Ortner 1995 : 191).

Nous voulons ainsi démontrer que les discours formulés par les Nehirowisiwok comptent tout autant que les contextes choisis pour transmettre et valoriser ces discours. Les concepts de créativité, d'imagination et d'agencéité (capacité d'agir, agency) sont ici centraux, tant dans l'élaboration du discours que dans les stratégies de transmission des savoirs et des identités (Holland et al. 2003; Clammer et al. 2004; Ortner 2006).

Les discours présentés ici sont tirés de trois contextes d'énonciation différents qui nous apparaissent tout à fait complémentaires et révélateurs de certaines stratégies de prise de parole déployées par les Nehirowisiwok. Nous nous basons sur des recherches de terrain réalisées depuis 2000 à Wemotaci dans le cadre d'une thèse de doctorat (Jérôme 2010a) et de travaux menés dans le cadre du projet Kinokewin (2006-2012, voir Poirier dans ce numéro). Ces présences sur le territoire atikamekw nehirowisiw, ainsi que le partage de nombreux événements culturels organisés par et pour les Nehirowisiwok, nous permettent de considérer différentes formes de transmission orale pratiquées au quotidien (semaines culturelles, activités rituelles, événements culturels...). C'est d'ailleurs à partir de ce travail que nous proposerons, dans la première section de ce texte, une réflexion sur la tradition orale des Nehirowisiwok et sur leur conception du territoire et du monde. Le renouvellement participatif (2010-2013) de l'exposition des Musées de la civilisation C'est notre
Histoire. Premières Nations et Inuit du XXI ${ }^{e}$ siècle constitue un second contexte d'énonciations de discours sur le Nitaskinan (voir Jérôme 2010b et 2013; La Boîte Rouge vif 2011, 2013 a et b, 2014). Lors de notre bref séjour de concertation dans la communauté de Manawan (mars 2011), les Nehirowisiwok ayant participé aux différentes activités ont majoritairement abordé le territoire comme thème central de ce qui devrait être présenté pour les Nehirowisiwok dans la nouvelle exposition qui allait être inaugurée en novembre 2013. Nous présenterons au fil du texte quelques récits tirés de cette concertation. À la différence $\mathrm{du}$ premier contexte (une recherche sur le long terme basée sur un partage du quotidien, révélant des discours et des conceptions fines du territoire), le second contexte permet de valoriser la construction de messages principalement destinés à des interlocuteurs non autochtones (les visiteurs du musée). Le contenu de ces messages reflète les revendications et les préoccupations actuelles de certains Nehirowisiwok à propos de leur territoire. Cet article s'appuiera enfin sur une troisième source, soit la production audiovisuelle atikamekw nehirowisiw du projet de formation aux techniques cinématographiques Wapikoni Mobile $^{2}$. Lanalyse des thèmes privilégiés par les jeunes réalisateurs nehirowisiwok ayant participé à ce projet permet non seulement de saisir différentes préoccupations de la jeunesse nehirowisiw à l'égard de leur territoire, mais également de voir comment ils convoquent et valorisent le discours de leurs aînés dans leurs productions. En partant $\mathrm{du}$ film Territoire des ondes (P. Boivin, 2006), nous présenterons quelques réflexions sur la place et l'importance des nouvelles technologies dans les processus de transmission des savoirs, d'affirmation d'une « identité atikamekw du territoire » (Poirier 2000: 143) et d'appropriation de médiums permettant de « dire » cette identité. Sur le plan théorique, il s'agit donc moins de proposer une réflexion sur le territoire que de documenter les discours sur celui-ci.

Cet article est organisé en trois parties. La première partie présente des éléments de la tradition orale des Nehirowisiwok et différentes figures de cette tradition orale. Le « dire » est ici celui d'une continuité, celui de la parole des ancêtres, celui qui fait référence au territoire raconté dans les grands récits, kitci atisokanak. Dans une seconde partie, nous valoriserons des discours évoquant les transformations du territoire, mais aussi le rôle de celui-ci dans les expériences de guérison. Ce « dire » est celui des aînés et des membres de la génération des pensionnats qui ont dû s'adapter aux nombreux changements ayant affecté l'utilisation et l'occupation du Nitaskinan et qui adressent leur message aux jeunes générations de Nehirowisiwok tout autant qu'à la société non autochtone. Nous terminerons cet article avec une troisième partie qui traitera des modalités d'appropriation des nouveaux médias par les autochtones, en particulier par les jeunes nehirowisiwok, afin d'exprimer leurs préoccupations et leurs perceptions à l'égard du territoire. Nous évoquerons différents projets qui montrent comment les nouveaux médias peuvent s'inscrire dans les stratégies de résistance autochtones, d'affirmation et de diffusion sur la scène publique de 
conceptions du monde et de savoirs locaux, renouvelant ainsi le rôle et la place actuels de l'art de raconter.

\section{MÉmOIRES DU TERRITOIRE : LES FIGURES ET LA CONCEPTION ATIKAMEKW NEHIROWISIW DU TERRITOIRE}

Avant de considérer les discours faisant état des transformations et des défis, il convient de livrer un regard sur les éléments de continuité. La tradition orale nehirowisiw comporte au moins trois grands types de récits de création du monde, chacun mettant en scène des personnages différents : le premier concerne la rencontre de l'espace et du temps, Nipinatcac et Kiwetinisiw; le second évoque la manière dont les choses, les animaux et les éléments (terre, eau, animaux, humanité, objets utiles à l'homme) se sont replacés après un déluge; le troisième parle de la création et de l'organisation de certains éléments de l'environnement à travers les péripéties de Wisaketcakw, le trickster atikamekw nehirowisiw. Ces trois grands types de récits, soit les kitci atisokanak, doivent être racontés à des moments précis, en fonction des saisons, du contexte de narration, du message à faire passer. Les kitci atisokanak se distinguent d'une autre forme de récit que l'on retrouve chez les Nehirowisiwok: les tipatcimowin. Ces récits se caractérisent par la présence de témoins directs ou indirects des événements ainsi racontés. Alors que les atisokanak sont intemporels, les tipatcimowin racontent des anecdotes qui se sont déroulées dans un passé plus ou moins proche.

Les Nehirowisiwok partagent certains traits avec leurs voisins cris (Eeyou), anichinabés (ojibwas, anishinaabeg) et innus, comme en témoignent Brightman et Brown (1988: 125) chez les Rock Crees, Hallowell chez les Anichinabés (1976 : 364-365) ou Vincent chez les Innus :

\begin{abstract}
Comme beaucoup d'autres peuples, comme les Nunamiut, comme les Tlingit, et les Athapascans, comme les plupart des Algonquins semble-t-il, les Montagnais reconnaissent deux catégories de récit. Les atanaukan rapportent la création du monde et les événements qui eurent lieu à l'époque où hommes et animaux n'étaient pas encore différenciés. Ils comprennent notamment le cycle du trickster Carcajou et racontent comment celui-ci donna leur forme définitive aux animaux, comment il nomma les arbres, comment naquirent les races, etc., donc comment ces divers éléments acquirent leurs caractères distinctifs. Nous classerions ces récits dans le genre mythe [...]. L'autre catégorie est nommée tipatshimun. [Celle-ci] contient d'une part des récits relativement fixés, dont on peut recueillir plusieurs variantes, d'autre part des discours ou commentaires qui le sont beaucoup moins et qui sont des créations personnelles récentes [...] (Vincent 1982 : 11-12)
\end{abstract}

Il est ici encore intéressant de noter la présence de la racine tipa-, qui exprime l'idée de compte, de modèle, de gestion. Tipatcimowin est également composé avec le morphème atcimo-, qui signifie, dire, raconter. Selon cette construction étymologique, tipatcimowin serait donc un récit dans lequel les événements sont rapportés fidèlement. José Mailhot et Sylvie Vincent ont entrepris de relever tous les termes innus composés avec la racine tipa-. Les auteures ont proposé l'interprétation suivante :

Les spécialistes de l'analyse des récits savent que les deux grandes catégories de récits qui existent dans les cultures algonquiennes correspondent à l'opposition entre TIPATSHIMU « il raconte une histoire, il rapporte une nouvelle » et ATANUTSHEU « il raconte une légende, un mythe ». La structure même du terme TIPATSHIMU confirme cette opposition car son sens littéral, d'après notre analyse, serait « il raconte en accord ou en correspondance (avec les faits) ». (Mailhot et Vincent $1980: 117$ et 118)

Ainsi, comme l'a soulevé Sylvie Vincent (1982), la différence entre les atisokanak et les tipatcimowin provient essentiellement de la manière dont le récit est rapporté :

Ce qui distingue les tipatshimun des atanukan, d'après les informateurs, c'est que les tipatshimun relatent des faits qui ont été vus et/ou vécus par des Indiens [...]. Lorsqu'ils racontent, les Montagnais d'ailleurs s'entourent de précautions verbales. Ils prennent soin de nommer le témoin de l'événement qu'ils rapportent ou la personne par laquelle le récit leur est parvenu. Ou bien ils signalent la présence des vestiges qui prouvent la véracité de leur dire. (ibid. : 12)

Transmettre les atisokanak est une préoccupation importante, « je trouve que c'est important parce que nos aînés s'en vont et c'est beaucoup d'histoires qui partent avec eux. C'est important pour moi et mes enfants, c'est notre histoire » (Jacques Newashish, mars 2011, Manawan) Comme un écho au titre de la nouvelle exposition des Musées de la civilisation, ces paroles d'un aîné nehirowisiw démontrent la place que doivent occuper les grands récits et les personnages qui y sont associés. Atcitcmoc, Amiskw, Masko, Kokotce, Cakwecic et Wisaketcakw en font partie. Wisaketcakw par exemple, est un héros comique que l'on retrouve également dans les cosmologies anichinabées (voir Servais 2005). À Opitciwan, la bande dessinée a été utilisée pour valoriser les péripéties de ce héros (Lachapelle 1982).

Si les péripéties de Wisaketcakw sont au cour des grands récits des Nehirowisiwok, les rencontres d'autres entités permettent de mieux comprendre certaines de leurs conceptions du monde et du territoire. Dans le récit qui suit, les points cardinaux, soit le Nord et le Sud, se rencontrent :

Shawinisi et Kiwetinisi étaient toujours ensemble et pourtant, ils ne s'entendaient pas très bien. Shawinisi portait tout le temps un sac plein d'oiseaux et Kiwetinisi voulait le lui voler. Alors un jour, Shawinisi donna à Kiwetinisi des perdrix, une pie et des hiboux, tous des oiseaux qui hivernent dans la région. Kiwetinisi n'était pas encore content et Shawinisi lui dit : "Tu devrais être content que je t'en donne autant! »Ce à quoi Kiwetinisi répondit : " Si tu ne m'en donnes pas plus, ce sera toujours l'hiver! » Mais Shawinisi n'écouta rien... Soudain, on entendit crier les femmes du village, car elles avaient vu un caribou traverser la rivière. Tous les hommes coururent aux canots et Shawinisi se joignit à eux, oubliant son sac derrière lui. Kiwetinisi ne perdit pas sa chance: dès qu'il vit Shawinisi monter dans un canot, il saisit le sac et l'éventra avec son couteau. Tous les oiseaux d'été étaient ainsi libérés. Kiwetinisi les dirigea vers les quatre points cardinaux : le Nord, le Sud, l'Est et l'Ouest. Dans ces temps-là, les carpes n'avaient pas de bouche. Une d'entre elles s'en fit une en se brûlant avec une éclisse de bois allumée. Ensuite, elle avertit Shawinisi du geste de son compagnon. Shawinisi tenta de revenir rapidement, mais le rat musqué avait mâché sa pagaie. Celle-ci se brisa et il ne put revenir à temps. C'est comme ça que l'été put recommencer ${ }^{3}$ !

La conception atikamekw nehirowisiw du monde, valorisée et transmise dans les grands récits, s'organise au 


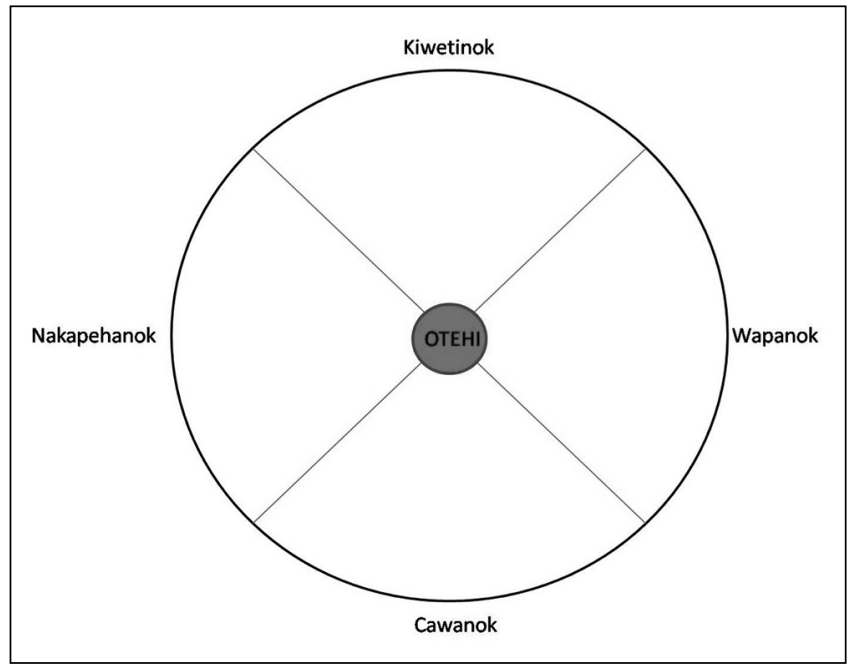

Figure 1

Tipahirotinan : points cardinaux

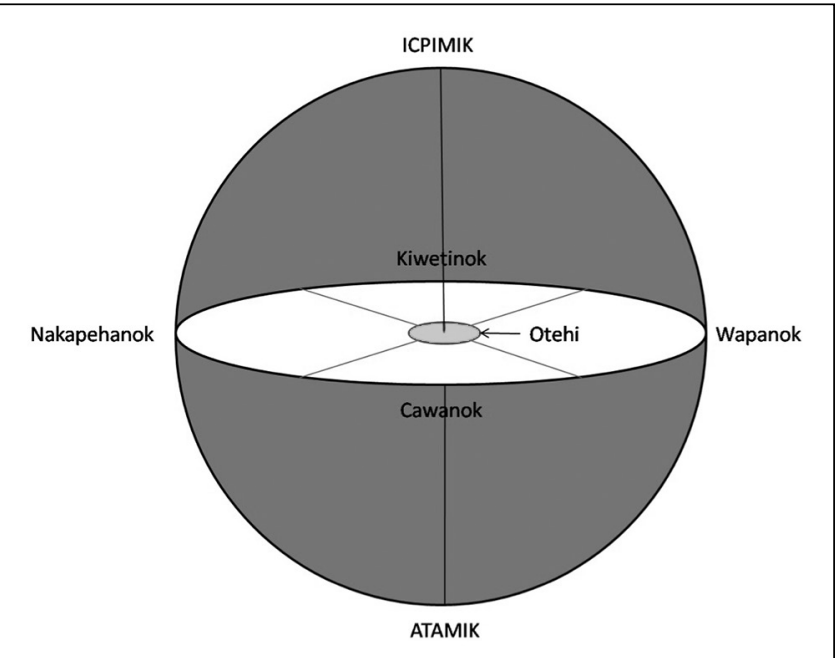

Figure 3

Sept directions

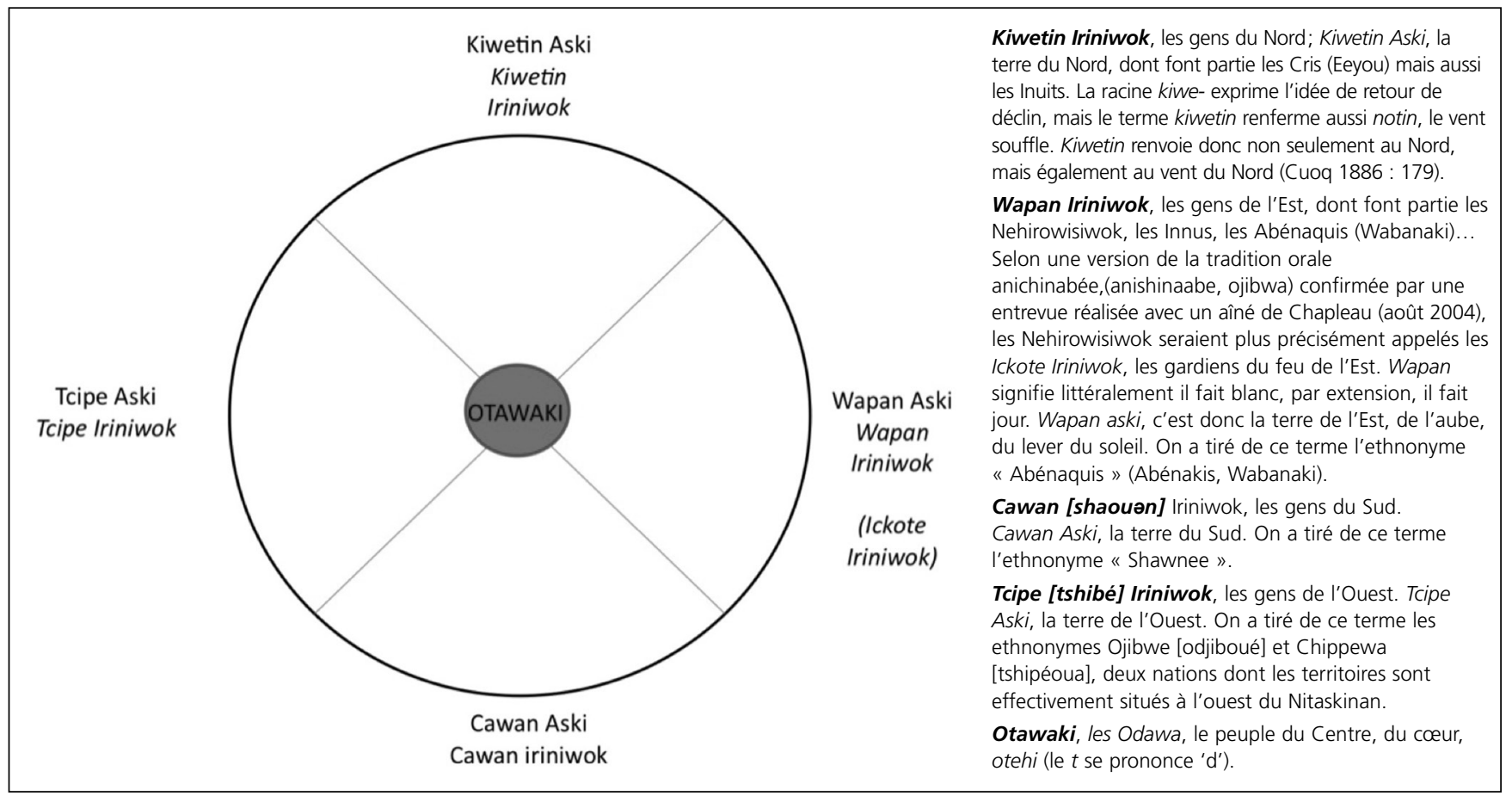

Figure 2

Territoires voisins

regard des points cardinaux (Tipahirotinan): le Nord (Kiwetinok), l'Ouest (Nakapehanok), le Sud (Cawanok) et l'Est (Wapanok) [fig. 1].

Il est intéressant de noter ici la proximité des termes tipahiskan (gestion du territoire, évoqué à différentes reprises dans ce numéro, voir Wyatt notamment) et tipahirotinan (points cardinaux). Ces deux termes sont construits avec la même racine, tipa-, qui renvoie à l'idée de mesure, de règle, de modèle, d'organisation (Cuoq 1886 : 402). Les points cardinaux apparaissent donc plus que comme de simples indicateurs de direction : c'est une manière de représenter la personne dans le monde et au cour de celui-ci (otehi). Cette conception du monde exprime également la position des Nehirowisiwok au regard des autres grands groupes autochtones de l'Amérique du Nord. Elle dépasse ainsi largement la question de la carte et des frontières établies à des fins politiques (voir fig. 2).

Ce territoire conçu au regard des quatre points cardinaux, avec un centre, otehi, ne suffit pas pour comprendre, ni pour rendre compte de toute la complexité de la conception atikamekw nehirowisiw du territoire. Les Nehirowisiwok considèrent également leur rapport en regard d'un monde du dessus, Icpimik (icpim-, en haut; -ik, dedans) et d'un monde du dessous, Atamik (atam-, au fond, sous, dedans ${ }^{4}$ ) [fig. 3].

Dans cette section, nous avons livré un aperçu de la manière dont les Nehirowisiwok pensent, conçoivent et transmettent leur identité territoriale. Les grands récits, 
racontés et transmis pendant les semaines culturelles de l'automne et du printemps, lors de cérémonies spécifiques (nouveau-né, loge à sudation, premiers pas...) ou encore lors d'événements marquant le quotidien (powwow, décès...), demeurent le support essentiel à la transmission de la conception nehirowisiw du territoire et du monde.

\section{LE TERRITOIRE COMME ESPACE POLITIQUE : DÉPOSSESSION ET TRANSFORMATIONS DE LA RELATION AU TERRITOIRE}

Qui es-tu? - Un homme. Je m'appelle Jacques Newashish. Enfin, c'est le nom qu'on m'a donné à ma naissance, au baptême: " Jacques ». Et "Newashish », le nom de mes ancêtres. Je suis Nehirowisiw - homme de la forêt - et je suis Atikamekw - c'est ma nation. (Jacques Newashish, mai 20135)

Dans sa réponse, Jacques Newashish distingue les conceptions atikamekw nehirowisiwok de l'identité des conceptions politiques. Comme nous l'avons précisé en introduction de ce numéro, le terme «Atikamekw » a été adopté dans les années 1970 pour des considérations politiques. Le terme « Nehirowisiw» est associé à l'univers forestier. Même chez les jeunes Nehirowisiwok dont le quotidien se construit au sein de l'espace de la communauté, le terme "Nehirowisiw » exprime un ensemble de savoirs liés au territoire et à l'univers forestier. Ernest Ottawa est décédé le 13 novembre 2011, quelques mois après que nous l'ayons rencontré dans le cadre du processus de renouvellement de l'exposition permanente. Président de la Nation atikamekw, membre fondateur du Conseil tribal de la Nation atikamekw, vice-président régional au Conseil Atikamekw-Montagnais, Ernest Ottawa a été négociateur en chef pour sa nation et a siégé au conseil de bande de Manawan comme conseiller pendant plus d'un mandat. Dans l'extrait présenté ci-dessous, il livre ses réflexions sur l'importance du territoire dans la vie actuelle et quotidienne des jeunes Nehirowisiwok. Il exprime un constat maintes fois entendu : les jeunes n'ont plus l'occasion de se rendre sur le territoire.

Nos jeunes se posent beaucoup de questions. Qui ils sont? Parce qu'il y a beaucoup de nos jeunes, on est tellement nombreux maintenant, les jeunes sont tellement nombreux, ils n'ont pas le temps. II y a l'école, ils ont leurs activités habituelles, sportives, $d^{\prime}$ autres activités, et puis ils n'ont pas le temps vraiment d'aller sur le territoire. Malgré qu'il y en a qui prennent le temps d'aller sur le territoire. Moi, ces jeunes-là qui veulent aller sur le territoire, je les encourage. Ils vont apprendre. Beaucoup. Bien plus que nous, possiblement. Moi, j'ai appris beaucoup de choses dans ma vie. À 11 ans, j'ai tué mon premier orignal. Après, j'ai été à la chasse beaucoup avec mes frères, [...] on allait souvent dans le bois, je les accompagnais. J'étais jeune à l'époque et je marchais vite en raquettes. Ils me disaient: "Ouvre la trail », et j'ouvrais la trail pour eux. Aussitôt qu'on voyait des traces d'orignaux, on savait qu'on pouvait avoir un ravage [...]. On pouvait tuer deux, trois orignaux. (E. Ottawa, mars 2011)

Lorsque Antoine Awashish, doyen actuel des Nehirowisiwok, parle du territoire, il évoque le rôle des femmes, les problèmes de comportement des jeunes, les processus d'apprentissage et les différences avec la vie d'aujourd'hui :

Durant notre enfance, il n'y avait rien qui nous dérangeait. [...] On nous a éduqués et nous avons reçu un enseignement sur la vie en forêt. II n'y avait rien d'autre que l'initiation au savoir sur la vie en forêt. On nous a enseigné à trapper, chasser et comment survivre en forêt. On accompagnait notre père quand il partait pour trapper ou quand il allait à d'autres endroits. En faisant cela, il nous enseignait. C'était ça l'enseignement qu'on recevait de la vie en forêt. De même pour la femme, elle enseignait à ses filles. Elle enseignait en quoi consistait son travail et ses activités. La femme avait aussi de nombreuses tâches qu'elle transmettait à ses filles et à ses enfants. [...] Les jeunes, garçons et filles, n'avaient pas de problèmes de comportement à l'époque. [...] Aujourd'hui, vous avez un magasin à votre disposition. Nous avons, quand même, survécu avec des produits de la chasse. N'importe quoi. Tout ce qu'on peut manger en forêt. C'est ce que nous mangions. Nous n'avions pas de magasin pour qu'on puisse manger quelque chose. Ça n'existait pas. C'est différent ce que vous faites aujourd'hui, et vous avez les moyens. Ce que vous avez aujourd'hui, nous n'avions pas ça dans mon temps. C'est tout ce que je peux vous raconter. (Antoine Awashish, doyen des Nehirowsiwok, film Notcimik Itekera, 2014)

Plusieurs films du Wapikoni Mobile mettent en avant une autre fonction du territoire dans les expériences : celle du mieux-être et de la guérison. Le film Don't Cry (2010) a par exemple été tourné sur le mont Tcikitnao, considéré comme un lieu de guérison et de ressourcement pour les gens d'Opitciwan. On y voit un groupe de jeunes femmes et de jeunes hommes chantant et jouant du tambour à main dans une forêt enneigée. Dans Masko Nimiwin (2008), Gilles Moar raconte comment la forêt lui a permis de mieux vivre le deuil de ses parents. Cette idée du territoire comme espace de ressourcement et d'apaisement est également évoquée dans Maskwesiwin Papiwin (La force du rire, 2011):

Quand je viens dans le bois, j'ai toujours une pensée pour ma grand-mère. Je voyais en elle une force, la persévérance, le courage, le respect, l'amour et la foi. J'aimais beaucoup aller dans le bois. On allait marcher pour le collet, la perdrix et pour aller chercher du sapin et des plantes médicinales [...] Pour moi, ma grandmère est une inspiration. Quand je vis des moments difficiles, je pense à elle et ça m'aide dans ma vie. (Jolène Chachai, film La force du rire, 2011)

Appuyé par de nombreux plans de la forêt et par le son des tambours et des chants des femmes, ce film insiste particulièrement sur la force féminine atikamekw nehirowisiw, force qui se construit à travers les relations que les femmes tissent entre elles, le ressourcement dans le bois, le souvenir des aînées et la thérapie, mais également par la valorisation des savoirs atikamekw nehirowisiwok, par les rituels tels que la tente de sudation et par le rire.

Lorsqu'on parcourt aujourd'hui le Nitaskinan, notamment à l'automne, il n'est pas rare de tomber sur des affiches révélant la difficile cohabitation entre les différents utilisateurs du territoire. Plantées sur les épinettes ou les bouleaux blancs, les inscriptions «Attention! chasseurs à l'affût », «Entrée interdite, propriété privée » ou « Territoire de chasse occupé » provoquent colère et frustration chez les Nehirowisiwok qui perçoivent ces avertissements comme une pratique supplémentaire d'appropriation du Nitaskinan. Nous avons pu constater trois formes de répliques : l'ignorance, l'action (enlever les inscriptions) et la dérision. Cette dernière forme de réplique correspond à une appropriation ironique de ce même dispositif technique: 
«Attention! territoire ancestral atikamekw ». Le ton est fidèle à l'humour atikamekw nehirowisiw: tourner en dérision un acte, une parole ou un événement ennuyant de sérieux ou, dans ce cas, rendre dérisoire un acte ressenti comme de la provocation. Lors d'une concertation organisée à Manawan en mars 2011, un groupe de discussion a été organisé conjointement par le Conseil de la Nation atikamekw, les Musées de la civilisation du Québec (MCQ) et La Boîte Rouge vif. La question de départ était relativement et volontairement simple: dans la nouvelle exposition des MCQ, que voudriez-vous adresser comme message? Les questions liées à la dépossession territoriale ont rapidement figuré au premier plan des discussions, révélant les préoccupations liées à l'utilisation, à l'occupation et à la gestion du territoire :

La déforestation, ça c'est un grand enjeu. On m'a même déjà dit " vous n'avez plus besoin d'orignal, plus besoin de castor parce que vous mangez des chops de lard maintenant! » Pourtant comme on l'a expliqué tantôt, les animaux aussi étaient médicamenteux, on a besoin des animaux pas seulement pour manger. Mais là, aujourd'hui, quand tu veux le manger, le castor, il est maigre parce qu'il n'a pas assez de nourriture... c'est ça l'enjeu. J'ai toujours lutté contre la déforestation. Pour montrer aux jeunes, ça nous prend la forêt. On a six saisons, ça prend le territoire pour montrer ça aux jeunes. Toutes nos technologies, nos savoirs sont reliés à la forêt. Pas de forêt, pas de chasse, pas de chasse, pas de connaissances. (Jérémie Chachai, mars 2011, Manawan)

Malgré des années de négociations et de revendications, les coupes forestières et les projets de construction de barrages hydroélectriques représentent encore aujourd'hui des sources de conflits importants. Si le processus de revendications canalise ces contentieux dans la sphère juridique, les enjeux, les défis et les désillusions liés aux règlements de ces contentieux touchent directement les familles et leur territoire. Pour illustrer ce point, nous prendrons l'exemple du conflit de l'été 2005 qui s'est créé autour d'un projet de construction de barrage. En 2002, le Conseil des Atikamekw de Wemotaci (CAW) signait une entente avec Hydro-Québec prévoyant la construction de deux barrages hydroélectriques (Rapides des Coeurs et Chute-Allard) sur la rivière Saint-Maurice (Tapiskwan), à quelques kilomètres de la communauté. Cette entente, comme de nombreuses autres signées par les Innus ou les Cris (Eeyou), prévoyait une compensation financière et l'embauche de travailleurs nehirowisiwok sur les chantiers de construction. Mais trois années et une élection locale plus tard (ayant entraîné un changement de dirigeants), certains membres des familles touchées dans l'utilisation et l'occupation du territoire familial se sont opposés à ces chantiers, faisant valoir l'absence de consultation, par l'ancien conseil de bande, à l'époque de la signature de l'entente. En quelques semaines, les contestations verbales ont glissé vers une opposition radicale matérialisée par deux tipis érigés à l'entrée de chacun des points d'accès aux chantiers de construction. Cet épisode, qui a pris fin à l'automne 2005, a montré toutes les difficultés liées à une conciliation des intérêts familiaux et communautaires dans ce type de projet. Si cette construction représentait une source importante de revenu pour les travailleurs de la communauté (sur trois années), elle entraînait également, selon les opposants, une modification importante de l'utilisation, la gestion et l'occupation du territoire pour les familles concernées.

Cet épisode de résistance a été abordé dans Le respect (2005). Ce film de Steven Chilton débute d'ailleurs avec la citation de César Newashish rapportée en ouverture du présent article. Pour la famille Chilton, cette résistance familiale peut devenir celle de tout un peuple, et c'est à ce type d'alliance, solidaire dans l'affirmation de droits distincts, que s'attendent les différents intervenants du film : "On espère susciter un effet d'entraînement chez les autres grandes familles de Wemotaci pour qu'elles aussi puissent se lever et affirmer leurs droits. »Cette revendication d'une autonomie politique et d'un contrôle sur la gestion du territoire atikamekw nehirowisiw s'inscrit dans une vision pacifiste et créatrice : "Il n'y aura pas de violence physique. Nous allons seulement parler. Pour ce faire, nous utiliserons notre pensée, notre parole et notre crayon, c'est tout. C'est le chemin à suivre pour avancer et savoir. » (Denis Chilton, film Le respect, 2005) Pour JeanMarc Niquay, Nehirowisiw de Manawan, les discussions avec les gouvernements devraient être réalisées selon une stratégie méthodique, mais devraient passer avant tout par une occupation et une utilisation soutenues du territoire afin que les gouvernements soient mis devant le fait accompli de la présence ancestrale et durable des Nehirowisiwok sur le territoire :

\begin{abstract}
[...] il y a cinq choses qui entreraient en ligne de compte. D'abord, la revendication, qui est pratiquement nulle. II y a la négociation, qui ne mène à rien, à moins d'avoir une bonne fenêtre. Malgré toutes les volontés politiques des politiciens, s'il n'y a pas de fenêtre, tu ne peux pas négocier. Après, il y a toute la question de I'utilisation. On I'utilise, le territoire. On l'utilise encore énormément. Au niveau culturel, pour aller camper, aller manger, aller parcourir. Et il y a l'occupation aussi, c'est important parce qu'il y a plusieurs camps, des Atikamekw de la région qui ont des camps partout sur le territoire. J'en ai même un. Puis, l'autre chose, c'est que les gouvernements devraient finir par comprendre qu'on est là et qu'on va toujours être là. II s'agit pour eux de s'adapter à nous parce que nous, on a essayé bien des années de s'adapter à eux, mais c'est à eux maintenant à s'adapter à nous. De là l'affirmation. L'affirmation nationale, l'affirmation de l'identité territoriale, de l'identité atikamekw, de l'identité culturelle, de toutes ces questions-là. (Jean-Marc Niquay, mars 2011, Manawan)
\end{abstract}

\section{« TERRITOIRE DES ONDES " : LE TERRITOIRE DANS LES NOUVEAUX MÉDIAS}

Quels rôles les nouveaux médias jouent-ils dans cette affirmation de l'identité atikamekw nehirowisiw du territoire (Poirier 2000 : 143)? Globalement, la prise du virage des technologies de l'information semble être une stratégie de choix pour s'assurer de la mise en valeur et de la vitalité des cultures autochtones, ainsi que de la transmission de savoirs à des jeunes évoluant dans la contingence d'une culture qui, avec les réseaux sociaux comme Facebook ou Bebo, les tablettes et les téléphones intelligents, devient désormais largement numérique (voir RAQ 42[1], 2012). Isuma TV permet par exemple aux Inuits de diffuser du contenu original, en langue inuktitut ou en anglais, qui 
correspond aux conceptions actuelles de l'identité inuite (voir à ce sujet Dupré 2010; Laugrand et Luna-Penna 2013). En général, cette expression identitaire à travers les médias contemporains semble dépasser les frontières régionales. Il s'agit d'une façon pour les jeunes autochtones de se faire du capital social à l'intérieur d'une culture globale, inscrivant leur démarche identitaire dans un esprit de socialisation et de partage avec le monde actuel. À propos de ce rayonnement, Alexander écrit : « ils [les Inuits] choisissent de partager le nouveau monde virtuel plutôt que de le préserver hors de la portée des non-Inuits » (Alexander et al. 2009 : 240). Laffirmation culturelle ne consiste pas, ici, à préserver une identité déjà existante, mais plutôt à la pousser plus avant, voire à la révéler par le processus même d'appropriation de l'espace numérique. Warschauer suggère ainsi qu'Internet puisse constituer, par sa virtualité, un excellent réseau exploratoire identitaire (Warschauer 1998 : 154). La possibilité d'y circuler de façon anonyme ou d'y embrasser des identités fictives permettrait aux jeunes utilisateurs de faire l'expérience de ce qui, en eux, est spécifique.

Tel qu'évoqué par Poirier dans ce numéro, l'équipe du projet Atikamekw Kinokewin s'est donné comme objectif le développement d'un site Internet destiné aux Nehirowisiwok. Valorisant des récits, des photographies, des vidéos, des témoignages, des travaux d'étudiants nehirowisiwok, des cartes toponymiques, des chants, des objets ethnologiques et des réflexions sur les activités traditionnelles, ce site Internet est présenté comme un outil pouvant favoriser, encourager ou encore compléter (et non remplacer) la transmission de certains savoirs atikamekw nehirowisiwok en lien avec le territoire. Étant donné le caractère sensible de certaines informations, ce site est en accès restreint depuis 2013. À l'été 2013, Vicky Veilleux a présenté à trois jeunes nehirowisiwok de Manawan différents onglets du site Internet Atikamekw Kinokewin, ainsi que quelques autres sites autochtones (www.nametauinnu.ca, www.fourdirectionsteachings.com, www.indiosonline.net), pour offrir des pistes de discussion sur l'importance des nouvelles technologies dans la mise en valeur des identités territoriales autochtones. Cette rencontre a permis d'identifier des dimensions jusque-là non explorées par l'équipe. En l'absence de téléphonie cellulaire dans la communauté, c'est l'Internet sans fil qui est aujourd'hui mis au service de la communication interpersonnelle de la majorité des jeunes, via leurs tablettes et téléphones cellulaires. Le réseau social Facebook est souvent la façon la plus efficace de communiquer avec quelqu'un. Les personnes qui n'ont pas de page Facebook manquent ainsi de nombreux événements importants dans la communauté : "Même les invitations aux mariages se font par Facebook. De nombreux aînés sont sur Facebook! » (Manawan, août 2013) Les discussions réalisées à propos du site Internet Atikamekw Kinokewin ont permis de confirmer la pertinence d'une telle ressource. Elles ont également soulevé quelques limites: "C'est bien notre culture qu'on voit à l'écran. C'est ça être Atikamekw. Mais il ne suffit pas d'être Atikamekw, il faut "vivre atikamekw" » (Manawan, août 2013). Pour ces jeunes, l'utilisation du site, en milieu scolaire notamment, devrait se coupler de sorties dans le bois, d'expériences concrètes. La création d'une application iPad pour le site Internet Atikamekw Kinokewin représenterait, selon ces jeunes, une voie de développement intéressante. Le groupe de tambour nehirowisiw Black Bear a d'ailleurs choisi cette option. Ce serait une façon d'attirer les jeunes vers le site. Cette réflexion fait écho au film de Patrick Boivin, Territoires des ondes (2006), dans lequel une réflexion est livrée sur l'utilisation des nouveaux moyens de communication pour affirmer les spécificités identitaires et culturelles nehirowisiwok:

La radio, la télévision, la vidéo, tout ça... même le journal, il faut qu'on utilise ces moyens-là pour pouvoir discuter avec le gouvernement. II faut absolument que ce soit vous qui disiez comment utiliser ces moyens de communication afin que le gouvernement comprenne les mœurs et coutumes des Atikamekw ou des autochtones. II faut qu'on aille chercher ces moyens pour pouvoir parler, discuter. Il faut absolument qu'on aille chercher ces moyens pour que nous puissions être écoutés et être forts, pour être ensemble dans notre démarche, si on veut que l'on parle de nous au gouvernement, de comment est-ce que nous, nous sommes encore attachés au territoire. C'est de cette manière que nous pourrons utiliser ces moyens, comme la radio et la télévision, pour que I'on puisse tous montrer, tous dire ce que nous avons, afin $q^{\prime}$ 'il y ait une prise de conscience, une prise de conscience communautaire de tous, une prise de conscience nationale en utilisant ces moyens-là. (Charles Coocoo, film Territoires des ondes, 2006)

Le projet Wapikoni Mobile ne se contente pas de donner la parole aux jeunes des Premières Nations, mais valorise leurs voix dans l'espace virtuel à travers son site Internet. Ces opportunités de se mettre en scène et de se raconter dans un monde virtuel permettent aux jeunes de prendre la parole dans des sociétés qui attribuent aux â̂nés le droit de raconter des histoires. L'affirmation identitaire est donc également celle d'une génération face à une autre, les règles qui régissaient traditionnellement la transmission orale, évoquées dans la première section de ce texte, étant ici renversées. C'est ce qu'a démontré l'étude de Wachowich et Scobie, un cyber-terrain de douze mois mené sur YouTube et ayant permis d'explorer les différentes formes d'interactions et de révélations de soi présentes dans les vidéos autoproduites par des jeunes Inuits.

\footnotetext{
L'art de raconter est une pratique cruciale dans les processus de reprise de pouvoir, puisque c'est à travers cette prise de parole que notre expérience passe de la conscience personnelle à la sphère publique. En faisant cela, nous ramenons ces expériences sous notre contrôle. En racontant des histoires, nous créons le monde autour de nous et devenons des êtres sociaux. (Wachowich et Scobie 2010 : 99)
}

Létude de cas de Singleton, Rola-Rubzen et al. - menée en Australie occidentale auprès de jeunes autochtones fréquentant un programme de développement des compétences liées aux technologies de l'information - révèle également le potentiel affirmatif d'une telle démarche d'appropriation de ces technologies. En effet, la maîtrise des moyens technologiques permettrait à la jeunesse de développer sa capacité d'agir (agency) [Singleton, RolaRubzen et al. 2009: 405], c'est-à-dire sa capacité à agir dans le monde. Lapparition des cultures autochtones au 
sein des médias technologiques peut être également lue comme une démarche de reprise de pouvoir de nations face à un monde global, les premières se rendant participantes d'un espace partagé où sont désormais visibles leurs préoccupations sociales, politiques et culturelles : «Cette approche sociopolitique de l'autonomisation mettant de l'avant le concept d'agencéité suggère un déplacement du pouvoir vers ceux qui en étaient dépourvus, leur permettant de faire émerger leurs préoccupations au cœur de l'agenda politique, social et culturel. » (ibid. : 406).

Internet et les nouveaux médias en général deviennent ainsi un nouvel espace de résistance autochtone, dans le sens de Ortner citée plus haut: Internet se pose comme une initiative supplémentaire, s'inspirant et nourrissant des initiatives et des projets existants. La réussite de l'entreprise de transmission via les technologies de l'information (ou d'autres médiums comme les bandes dessinées) semble résider dans le processus même de construction de ces nouveaux espaces. Deux éléments semblent ici primordiaux: l'autodétermination et le respect des ontologies locales $^{6}$. Par autodétermination, nous entendons l'engagement des acteurs concernés dans chacune des étapes du projet, ainsi que la détention par eux du principal pouvoir décisionnel. L'exemple du processus de création du jeu Digital Songlines, outil multi-média basé sur la transmission orale de la culture aborigène australienne, illustre bien cette philosophie. En effet, la vision du projet, qui était une collaboration entre un centre de recherche et différentes communautés aborigènes australiennes, s'articule en ces termes: « ... offrir un véhicule de partage du savoir culturel approprié et abordable qui soit la propriété de la communauté, géré par cette dernière et orienté vers ses besoins » (Leavy et al. 2007 : 162). Tous les enjeux étaient discutés à l'intérieur d'une démarche de consultation cyclique avec l'ensemble des communautés engagées dans le processus de développement du projet. L'élaboration de protocoles et de lignes directrices éthiques aurait également aidé à établir des procédures basées sur le respect et la transparence (ibid. : 163). Dans le même sens, à la suite de son expérience autour de la conception du CD-ROM Dream Tracker avec les Walpiris d'Australie, Glowczewski écrit sur le désir légitime de ces derniers de « décider de ce qui peut être rendu public ou non sur le Net» (Glowczewski 2009 : 84). L’autodétermination n’agit donc pas seulement sur la nature du processus de production du nouveau média, mais également sur la sélection du contenu qui sera diffusé. Le respect de la propriété intellectuelle, le respect des sensibilités - par exemple autour du «tabou des morts» (ibid. : 73) - et le discernement quant aux données qui doivent demeurer secrètes et celles qui peuvent être rendues publiques sont des enjeux d'une importance capitale. Linvestissement du monde virtuel peut également être une façon de répondre à l'information inappropriée véhiculée au sujet des communautés autochtones, leur permettant ainsi de regagner du contrôle et une certaine cohérence sur l'identité vécue et véhiculée (voir à ce sujet Iseke-Barnes 2002). Les sites Internet, les productions audiovisuelles et les nouvelles technologies en général valorisent et favorisent les processus atikamekw nehirowisiwok de résistance et d'affirmation identitaire et culturelle qui s'inscrivent dans un continuum avec des actions, des négociations et des revendications concrètes. La production du discours, quel que soit le contexte d'énonciation, ne suffit pas à rendre efficace ce qu'il véhicule. Elle doit s'accompagner de stratégies de diffusion et de valorisation

\section{Conclusion}

En ouvrant sa célèbre citation par Witamowikok, « Dites-leur », César Newashish indique une voie privilégiée de résistance et d'affirmation de la souveraineté des Nehirowisiwok: celle du discours. Les différentes prises de parole autour du territoire rapportées dans cet article vont dans le sens de cette invitation de César Newashish. Elles sont autant d'expressions vivantes du projet d'autodétermination et de souveraineté des Nehirowisiwok, qui a trouvé son apogée en septembre 2014 avec l'affirmation de la souveraineté des Nehirowisiwok sur le Nitaskinan (voir l'introduction de ce numéro), largement relayée sur les réseaux sociaux et dans l'espace médiatique. Ces déclarations politiques, les récits de la tradition orale, les activités pratiquées dans le bois, la lutte contre la dépossession et la déforestation ou la continuité d'une transmission de l'identité atikamekw nehirowisiw et des savoirs liés à l'univers forestier via les nouveaux médias, sont autant de démarches qui s'entrecroisent et participent conjointement à la construction de la relation que les Nehirowisiwok entretiennent avec leur territoire.

La vingtaine de films de la production de Wapikoni Mobile et les différents projets dans le cadre desquels les Nehirowisiwok prennent la parole illustrent bien cette multiplicité et cette complémentarité des différentes façons atikamekw nehirowisiwok de dire le territoire. Les thèmes (voir plus loin l'encadré des résumés de films produits par Wapikoni) abordés dans les films de Wapikoni Mobile traduisent les préoccupations et les perceptions actuelles des Nehirowisiwok à propos de leur territoire : le territoire comme lieu de guérison et de ressourcement, de négociations et de revendications, de mémoire et d'histoire. Leur diffusion sur une plateforme Internet en accès ouvert, les bandes dessinées et les livres jeunesse utilisés comme médium de diffusion de certains récits ou les applications interactives développées par les groupes de joueurs de tambour renforcent cette idée de résistance créative et constructive et d'affirmation de la souveraineté des Nehirowisiwok sur leur territoire. Même si elles ne s'intègrent pas dans un mouvement politique institutionnalisé de résistance, comme a pu l'être par exemple l'American Indian Movement, les différentes stratégies de prise de parole privilégiées par les Nehirowisiwok prennent une forme de résistance qui s'exprime à travers la volonté de rétablir des relations entre les générations, avec les autres nations, mais aussi avec les non-autochtones. Les concepts de relations, de transmission et d'apprentissage apparaissent aujourd'hui comme des concepts clés qui favorisent la continuité, les innovations et la mise en valeur de certains savoirs et de certaines pratiques. Dans tous ces projets, y compris dans le projet de renouvellement de l'exposition permanente des Musées 
de la civilisation de Québec pour lequel de nombreux Nehirowisiwok ont « pris la parole » afin d'expliquer leur vision de l'identité nehirowisiw du territoire, les discours semblent être les mêmes depuis le début du processus de revendication: colère, frustration et découragement devant les actions de dépossession territoriale, rendant la déclaration de César Newashish toujours aussi pertinente vingt ans après qu'elle a été prononcée. Si ces différentes stratégies de prise de parole sont une occasion pour les plus jeunes générations de se positionner comme des acteurs des stratégies actuelles d'affirmation identitaires, elles permettent également aux membres des générations précédentes d'adresser leur propre message aux jeunes qui sollicitent leurs points de vue dans les films qu'ils réalisent. Le territoire évoqué est cette fois celui de leur propre vie, celui qui se construit dans la résistance au quotidien (Scott 1985) :

\begin{abstract}
Le message que je souhaite envoyer aux jeunes, c'est de regarder en avant, vers l'avenir. Cherchez ce qui vous rend épanouis. Peut-être allez-vous rencontrer des obstacles qui vont vous empêcher de réaliser vos rêves.[...] Soyez forts. Dès que vous savez ce que vous voulez faire dans la vie, cherchez les ressources qui peuvent vous aider à réaliser votre rêve. [...] Quand vous vivrez pleinement dans votre rêve, vous regarderez derrière et vous direz: " je suis responsable de mon bien-être ». II faut renverser I'histoire. [...] Vous serez une jeunesse pleine de fierté. Ce que je souhaite vous dire, c'est que l'avenir vous appartient, c'est à vous de décider ce qu'il sera. Soyez forts, entraidez-vous et prenez les décisions pour vous-mêmes. J'ai hâte de vous voir, parce que vous êtes la relève, [...].Voilà ce que je voulais vous dire. (Mary Coon, film La relève, 2010, Wapikoni Mobile).
\end{abstract}

\section{Notes}

1. Projet du CRSH, Territorialités autochtones postcoloniales, transmission et autonomie. La Nation atikamekw et l'univers forestier.

2. Wapikoni Mobile est un projet ambulant de formation aux techniques cinématographiques, né en territoire atikamekw nehirowisiw et qui fête ses dix ans en 2014. Voir <www.wapikoni.ca> et le texte de Manon Barbeau sur la genèse du projet, dans ce numéro.

3. Conteur: Peter Neashit, Opitciwan, 22 août 1927, archives CNA et site Internet <www.manawan.org >

4. Atamitch, dedans, au fond (Silvy 1974: 20); Anâm, anami, under, underneath, beneath /anâmaking, sous la surface de la terre (Barraga 1992: 27-28); Anam, sous, au fond, dedans (Cuoq 1886: 40).

5. Entrevue de Jacques Newashish, <http://www.feever.be/ jacques-newashish> (consulté le 14 janvier 2014).

6. Le concept d'ontologie locale est ici défini comme un ensemble de savoir-faire et de savoir- être constituant une manière spécifique de concevoir le monde et les relations entre ou avec les entités qui le composent.

\section{Remerciements}

Cet article n'aurait pu être possible sans le financement du Conseil de recherches en sciences humaines du Canada (CRSH), projet Territorialités autochtones postcoloniales, transmission et autonomie. La Nation atikamekw et l'univers forestier, 2012-2015, et du Fonds de recherche-Québec, Société et culture (FRQSC), projet Savoirs, territoires et réseaux autochtones : la relation ville-communauté dans les expressions contemporaines des identités autochtones, 20132016. Nous tenons à remercier Sylvie Poirier, Charles Coocoo,
Christian Coocoo ainsi que les deux évaluateurs anonymes d'une première version de ce texte pour leur lecture attentive et leurs judicieux commentaires.

\section{Ouvrages cités}

ALEXANDER, Cynthia, J., A. ADAMSON, G. DABORN, J. HOUSTON et V. TOOTOO, 2009 : «Inuit Cyberspace: The Struggle for Access for Inuit Qaujimajatuqangit». Journal of Canadian Studies/Revue d'études canadiennes 43(2) : 220-249.

BARAGA, Frederic, 1992: A Dictionary of the Ojibway Language. Minnesota Historical Society Press, Minnesota.

BLEIKER, Roland, 2000: «Writing Human Agency After the Death of God », in Louise Amoore (dir.), The Global Resistance Reader: 92-98. Routledge, London.

BRIGHTMAN, Robert, et J. BROWN, 1988: The orders of the Dreamed. George Nelson on Cree and Northern Ojibwa Religion and Myth, 1823. The University of Manitoba Press, Winnipeg.

CLAMMER, John, S. POIRIER et É. SCHWIMMER, 2004 : Figured Worlds: Ontological Obstacles in Intercultural Relations. University of Toronto Press, Toronto.

CUOQ, Jean-André, 1886 : Lexique de la langue algonquine. J. Chapleau, Montréal.

DUPRÉ, Florence. 2010 : «IsumaTV, les enjeux virtuels d'un réseau d'artistes inuit - Entretien avec Stéphane Rituit». Cahiers du CIÉRA 5 : 17-27.

FOUCAULT, Michel, 2005: « Method » in Louise Amoore (dir.), The Global Resistance Reader : 86-91. Routledge, London.

GAGNÉ, Natacha, et M. SALAÜN, 2010 : Visages de la souveraineté en Océanie. L'Harmattan, Paris.

GLOWCZEWSKI, Barbara, 2009: "Chapitre 4 - Restitution de données anthropologiques en multimédia: défis pratiques, éthiques et scientifiques », in C. Albaladejo et al. (dir.), La mise à l'épreuve: le transfert des connaissances scientifiques en questions: 69-85. Éditions Quae, Coll. Update Sciences \& Technologies.

HALLOWELL, Alfred I., 1976 : « Ojibwa Ontology, Behavior and World View », in A.I. Hallowell (dir.), Contributions to Anthropology, Selected Papers of A.I. Hallowell : 357-390. University of Chicago Press, Chicago.

HOLLAND, Dorothy, et al., 2003 [1998] : Identity and Agency in Cultural Worlds. Harvard University Press, Cambridge.

JÉRÔME, Laurent, 2010a: Jeunesse, musique et rituel chez les Atikamekw (Haute-Mauricie, Québec) : ethnographie d'un processus d'affirmation identitaire et culturelle en milieu autochtone. Thèse de doctorat, département d'anthropologie, Université Laval. <http://ariane.ulaval.ca/cgi-bin/recherche.cgi?qu=a2006239> (consulté le 2 octobre 2014).

—, 2010b: «Vers une nouvelle exposition au Musée de la civilisation ». Recherches amérindiennes au Québec 40(1-2) : 161-163.

—, 2013: «Cosmologies autochtones et musée: diversité des expériences et des points de vue dans un processus de consultation ». Histoire, monde et cultures religieuses 27 : 19-42.

LA BOÎTE ROUGE VIF, 2011 : Atelier créatif. Manawan.

—, 2013a : Atelier de conception "Espace". Parcours de l'exposition. Québec.

—, 2013b : Portefolio : La Boîte Rouge vif. Chicoutimi. <http://www. uqac.ca/design/wp-content/uploads/2013/07/BRV_crew.pdf>, le 6 février 2014.

LA BOÎTE ROUGE VIF et LE MUSÉE DE LA CIVILISATION, 2014: Parcours des mondes autochtones. <http://www.mondesautochtones.ca/> (consulté le 26 mars 2014).

LAUGRAND, Frédéric, et Galo LUNA-PENNA, 2013 : «Isuma.tv ou la Babel du Grand Nord : religions, images autochtones et médias électroniques». Recherches amérindiennes au Québec $43(2-3): 31-47$ 
LEAVY, Brett et al., 2007 : «Evaluating the Digital Songlines Game Engine for Australian Indigenous Storytelling », in Proceedings of the 13th International Conference on Virtual Systems and Multimedia : 162-171. International Society on Virtual Systems and Multimedia, Brisbane, Queensland, Australia. <http:// resources.ethnosproject.org/wp-content/uploads/digital_ songlines_leavy.pdf> (consulté le 15 septembre 2014).

MAILHOT, José, et S. VINCENT, 1980 : Le discours montagnais sur le territoire. Rapport soumis au Conseil attikamek-montagnais.

ORTNER, Sherry B., 1995: «Resistance and the Problem of Ethnographic Refusal ». Comparative Studies in Society and History 37(1) : 173-193.

—, 2006: Anthropology and Social Theory. Culture, Power and the Acting Subject. Duke University Press, Durham.

POIRIER, Sylvie, 2000 : «Contemporanéités autochtones, territoires et (post)colonialisme ». Anthropologie et Sociétés 24(1): $137-153$

SERVAIS, Olivier, 2005 : Des jésuites chez les Amérindiens ojibwas histoire et ethnologie d'une rencontre, $X V I I{ }^{e}-X X^{e}$ siècles. Éditions Karthala, Paris.
SCOTT, James C., 1985 : Weapons of the Weak, Everyday Forms of Peasant Resistance. Yale University Press, New Haven.

—, 1990 : Domination and the Arts of Resistance: Hidden Transcripts. Yale University Press, New Haven.

SINGLETON, Rola-Rubzen, et al., 2009 : " Youth empowerment and information and communication technologies: A case study of a remote Australian Aboriginal community». GeoJournal 74(5) : 403-413.

SILVY, ANTOINE, 1974 : Dictionnaire montagnais-français. Presses de l'Université du Québec, Montréal.

VINCENT, Sylvie, 1982: «La tradition orale montagnaise. Comment l'interroger? » Cahiers de Clio 70 : 5-26.

WARSCHAUER, Mark, 1998: «Technology and Indigenous Language Revitalization: Analyzing the Experience of Hawai'i ». The Canadian Modern Language Review/La Revue canadienne des langues vivantes 55(1) : 139-159.

WACHOWICH, Nancy, et W. SCOBIE, 2010 : « Uploading selves: Inuit digital storytelling on YouTube». Études/Inuit/Studies 34(2) : 81-105.

\section{Le Nitaskinan dans le projet Wapikoni Mobile*}

\section{Nimocom otaski (La forêt de mon grand-père)}

http://www.wapikoni.ca/films/nimocom-otaski-la-foret-de-mongrand-pere

Réalisation : Sonia Chachai

Année de production : 2004

Durée : 19:30 min.

Communauté : Opitciwan

Dans Nimocom otaski, Ronny Chachai mène une enquête sur l'exploitation forestière et a choisi le thème de la résistance face à la déforestation, plus spécifiquement celle de la famille Chachai et d'autres Nehirowisiwok d'Opitciwan face aux coupes intensives effectuées sur leurs territoires par la compagnie forestière BarretteChapais. Le territoire est abordé à la fois comme lieu de mémoire, comme source de vie et comme élément déterminant de l'identité nehirowisiw. Ici, la préservation de l'univers forestier est clairement associée à la continuité de la culture autochtone: "C'est toute la culture autochtone qui risque de se perdre. [...] Je vais faire ce que je peux pour ralentir la déforestation parce que c'est l'essence de notre identité qui se trouve dans cette forêt. » (Ronny Chachai)

\section{Le respect}

http://www.wapikoni.ca/films/le-respect

Réalisation : Steven Chilton

Année de production : 2005

Durée : 8:21 min.

Communauté : Wemotaci

Le respect aborde l'acte de résistance posé en 2005 par la famille Chilton, qui désirait se faire entendre et affirmer ses droits sur son territoire familial, lequel était visé par des projets d'Hydro-Québec (barrages hydroélectriques de Rapides des Cœurs et Chute Allard). Plusieurs intervenants dans le film expriment leur souhait de voir émerger un mouvement d'affirmation solidaire et de faire de cette résistance familiale celle de tout un peuple : "On espère susciter un effet d'entraînement chez les autres grandes familles de Wemotaci pour qu'elles aussi puissent se lever et affirmer leurs droits ». Le film s'ouvre sur la citation de César Newashish : "Dites-leur que nous n'avons jamais cédé notre territoire, que nous ne l'avons jamais vendu, que nous ne l'avons jamais échangé, de même que nous n'avons jamais statué autrement en ce qui concerne notre territoire. » (1994)

\section{Tikinakan}

http://www.wapikoni.ca/films/tikinakan

Réalisation : Alexandra Awashish, Carole-Anne Niquay

Année de production : 2006

Durée : 6:49 min.

Communauté : Wemotaci

Ce court métrage présente la fabrication, I'histoire et l'origine du tikinakan (porte-bébé), un objet fabriqué à partir des ressources du territoire et participant à la transmission de l'identité atikamekw nehirowisiw. L'enfant placé dans le dos de sa mère se trouve face au territoire, et c'est en le voyant défiler qu'il apprend d'où il vient. Cette référence au territoire en tant que lieu d'origine montre comment l'identité des Nehirowisiwok, qui peut également se lire à travers des objets comme le tikinakan, est indissociable de la notion de territoire.

\section{Territoire des ondes}

http://www.wapikoni.ca/films/territoire-des-ondes

Réalisation : Alland Flamand, Patrick Boivin

Année de production : 2006

Durée : 10:46 min.

Communauté : Wemotaci

Les cinéastes mettent en parallèle les médiums de communication qu'utilisaient leurs ancêtres et ceux qui sont utilisés aujourd'hui. C'était le tambour rassembleur (tewehikan) qui était utilisé autrefois pour communiquer. Moyen de communication moderne, la radio permet tout autant de resserrer les liens dans la communauté. Selon Charles Coocoo, un aîné de la communauté de Wemotaci, la radio et les autres moyens de communication comme la télévision et le journal, peuvent être une façon d'affirmer l'identité collective et individuelle. II voit l'opportunité d'élargir cette prise de conscience à

* On pourra aussi consulter Recherches amérindiennes au Québec 35(3), 2005, qui était accompagné du DVD «Paroles de jeunes autochtones » regroupant quelques-uns des documents réalisés par le projet Wapikoni Mobile lors de sa première année. [NDLR] 
l'échelle nationale afin de pouvoir être vus et entendus par le gouvernement. Les communications, en tant qu'espaces d'expression de I'attachement que les Nehirowisiwok portent à leur territoire, deviennent alors le véhicule solidaire des revendications territoriales des Nehirowisiwok.

\section{Kice iriniw acitc sipiriw (Le vieil homme et la rivière)}

http://www.wapikoni.ca/films/kice-iriniw-acitc-sipiriw-le-vieilhomme-et-la-riviere

Réalisation : Steven Chilton

Année de production : 2006

Durée : 5:09 min.

Communauté : Wemotaci

Ce film d'animation met en perspective la relation vécue au territoire, via des activités (canot, pêche, portage), la présence animale (traces d'orignal, aigle, poissons, huard) et l'occupation humaine. Surtout, il rend compte de la relation au grand-père (nimocom) et des processus de transmission intergénérationnels des savoirs liés au territoire. C'est à travers le lien avec le grand-père que se forge la relation au territoire et que naît la conception atikamekw nehirowisiw de celui-ci.

\section{Kitaskino (Notre territoire)}

http://www.wapikoni.ca/films/kitaskino-notre-territoire

Réalisation : Henman W. Niquay

Année de production : 2007

Durée : 4:53 min.

Communauté : Manawan

Kitaskino présente une discussion entre un grand-père (Dan-Georges Niquay) et son petit-fils (Herman W Niquay). L'aîné parle de la nécessité de protéger la forêt en tant que ressource alimentaire, curative et identitaire. L'humain vit en interdépendance avec le territoire, en étant à la fois tributaire et protecteur. L'idée d'intégrité du territoire est, encore une fois, associée à l'idée de continuité culturelle (l'utilisation du territoire étant une dimension centrale de cette dernière) : " J'admire beaucoup que tu aies fait avancer le dossier des aires protégées. [...] Pour que ça fonctionne, pour qu'on puisse poursuivre notre culture, l'éducation des générations futures, comment la nation atikamekw nehirowisiw vit avec le territoire. »

\section{Notcimi}

http://www.wapikoni.ca/films/notcimi

Réalisation : Pinaskin Ottawa

Année de production : 2007

Durée : 5:20 min.

Communauté : Manawan

Chanson en atikamekw nehirowisiw exprimant les sentiments d'impuissance, de colère et de tristesse de l'auteur-interprète Pinaskin Ottawa. Les images mettent en opposition un territoire intact (lac, forêt, canot, artisanat) et un territoire altéré (coupes à blanc). Les paroles soutiennent cette opposition entre le territoire d'hier, celui des grands-pères, et le territoire d'aujourd'hui, marqué par la dépossession et la destruction.

\section{Wapimatan}

http://www.wapikoni.ca/films/wapamitan

Réalisation : Léa Naukie Dubé et l'équipe du Wapikoni Mobile

Année de production : 2008

Durée : 2:26 min.

Communauté : Opitciwan

Des jeunes filles livrent une performance chantée sur le bord d'un lac Comme c'est le cas dans plusieurs autres films de Wapikoni Mobile, les images qui accompagnent les performances chantées (ou dansées) présentent un espace forestier harmonieux. C'est à cette image du territoire intact, préservé, perçu comme authentique, que l'on rattache l'identité atikamekw nehirowisiw. Dans Wapimatan, comme dans d'autres productions, les réalisateurs associent ainsi l'intégrité de
I'univers forestier à la force tranquille d'une société et d'une culture toujours résilientes.

\section{Masko nimiwin (La danse de l'ours)}

http://www.wapikoni.ca/films/masko-nimiwin-la-danse-de-lours Réalisation : Marie-Christine Petiquay

Année de production : 2008

Durée : 6:23 min.

Communauté : Manawan

C'est dans le bois que Gilles Moar est allé vivre le deuil de son père. Une plume trouvée lors de cette retraite lui permit de trouver la guérison (miromatisiwin) qu'il recherchait. II nomma sa fille du nom de cette plume, Mikona. Lors de ce séjour dans le bois, il a également compris que jouer du tambour pourrait lui permettre de retrouver équilibre et harmonie dans sa vie. Les animaux qu'il a pu observer l'inspirent dans sa pratique de la danse. Masko nimiwin montre un homme qui fait de sa démarche artistique et culturelle une façon de ritualiser sa relation au territoire et de transmettre cette relation. Le film est tourné au powwow annuel de Manawan.

\section{Notcimik itekera}

http://www.wapikoni.ca/films/notcimik-itekera

Réalisation : Eden Awashish

Année de production : 2009

Durée : 6:43 min

Communauté : Opitciwan

Antoine Awashish, aîné d'Opitciwan, raconte le mode de vie d'autrefois. "Durant notre enfance, il n'y avait rien qui nous dérangeait. [...] On nous a éduqués et nous avons reçu un enseignement sur la vie en forêt. II n'y avait rien d'autre que l'initiation au savoir sur la vie en forêt. » I| parle de la transmission intergénérationnelle des savoirs liés à ce mode de vie, du rôle qu'avaient l'homme et la femme nehirowisiwok dans ce système d'interdépendance. II met en contraste la nécessité d'entretenir une relation intime avec le territoire pour vivre et la grande facilité avec laquelle on se procure aujourd'hui la nourriture dans les magasins.

\section{Thunderbirds Junior}

http://www.wapikoni.ca/films/thunderbirds-junior

Réalisation : Équipe du Wapikoni Mobile

Année de production : 2009

Durée : 4:54 min

Communauté : Opitciwan

Le groupe Thunderbirds Jr joue du tambour de powwow (tewehikan) et chante une de ses compositions dans la communauté d'Opitciwan. Prises d'un bateau en mouvement sur un lac, les images choisies pour accompagner la performance des joueurs dressent un parallèle entre l'acte musical et la relation vécue au territoire. Le chant et le jeu de tambour sont vus comme une démarche d'affirmation identitaire, démarche ancrée dans ce territoire vécu.

\section{Don't cry}

http://www.wapikoni.ca/films/dont-cry

Réalisation : Allen Clary, Danicka Dubé, Dayla Awashish,

Ken Awashish

Année de production : 2010

Durée : 5:09 min

Communauté : Opitciwan

Ce vidéoclip mettant en scène de jeunes joueurs de tambour à main a été tourné sur le mont Tcikitanaw (la plus haute montagne), un lieu de ressourcement important pour les Nehirowisiwok d'Opitciwan, durant I'hiver 2010. Cette reprise d'un chant du groupe Eyabay (Red Lake, Minnesota) est illustrée par les images d'un territoire recouvert de neige évoquant l'idée d'un lieu pur, immaculé, blanc, hors du temps. Les paroles du chant soutiennent cette idée d'un endroit hors du monde, porteur d'espoir et de renouveau : "It's not there in this 
world. I wanna know to be there, all day new. Don't cry, close your eyes. And I will be right there, all day new. »

\section{Ken Awashish - Opitciwan}

http://www.wapikoni.ca/films/ken-awashish-opitciwan

Réalisation : Ken Awashish

Année de production : 2010

Durée : 2:29 min.

Communauté : Opitciwan

Ken Awashish, jeune tambourineur, interprète un chant de sa composition exprimant son attachement à sa communauté, Opitciwan. Cette célébration d'Opitciwan et du territoire qui l'entoure par le chant et le jeu de tambour à main est réalisée en langue atikamekw nehirowisiw.

\section{La relève}

http://www.wapikoni.ca/films/releve

Réalisation : Bobby Papatie

Année de production : 2010

Durée : 3:59 min.

Communauté : Wemotaci

Dans La relève, l'aînée Mary Coon adresse un message d'avenir et d'espoir à la jeunesse de Wemotaci. Elle lui parle de force, d'entraide, d'autodétermination et de rêve : "L'avenir vous appartient, c'est à vous de décider ce qu'il sera. Soyez forts, entraidez-vous et prenez les décisions pour vous-mêmes. " Le film alterne entre des images du territoire, du réalisateur/chanteur/joueur de tambour Bobby Papatie, d'un danseur de powwow et de jeunes nehirowisiwok dans leur communauté. Cette alternance vient ancrer le message d'avenir de Mary Coon dans un lien entre univers forestier et territoire social.

\section{Maskwesiwin Papiwin (La force du rire)}

http://www.wapikoni.ca/films/maskwesiwin-papiwin-la-force-du-rire Réalisation : Jolène Chachai, Stella Chachai

Année de production : 2011

Durée : 9:17 min.

Communauté : Opitciwan

Supporté par des images du territoire et par le son des tambours et des chants féminins, ce film laisse toute la place à la complicité des femmes. Ces dernières, en préparant de la bannique, de la viande et des beignes, parlent de ce qui les rend fières d'être femmes, de ce qui leur donne force et énergie. Le film présente en filigrane la relation au territoire et à différentes activités (collets, plantes médicinales, préparation de la nourriture, confection de mitaines et de mocassins en cuir) en tant que voie de guérison et d'affirmation de la force féminine. Cette force, à laquelle on fait référence maintes fois durant le film, se construit à travers le ressourcement (dans le bois, dans le souvenir des aînées et par la thérapie), par le recours aux savoir-faire et aux rituels tels que la tente de sudation, et par le rire : "Quand on est entre femmes, ça nous aide à nous ressourcer, à rire et à devenir plus fortes. La force du rire. »

\section{Kokominokw ki witamakonohok (L'héritage de nos grands-mères)}

http://www.wapikoni.ca/films/kokominokw-ki-witamakonohok-lheritage-de-nos-grands-meres

Réalisation : Claudie Ottawa

Année de production : 2011

Durée : 4:43 min.

Communauté : Manawan

Cette fiction met en scène les défis liés à la transmission de l'héritage culturel nehirowisiw dans un contexte de disparition des aînés. Le film évoque le deuil vécu par des jeunes femmes à la suite de la mort d'une aînée, mais aussi le deuil d'un savoir culturel qui pourrait, en partie, s'éteindre avec elle. D'où les dernières phrases du film, invitant l'auditeur (nehirowisiw) à marcher sur les pas des aînés, à perpétuer I'héritage culturel, à retourner aux sources: "Nos aînés ont vécu. Marchons ensemble sur leurs traces. Il est de notre devoir de perpétuer notre héritage culturel. Retournons aux sources. »

\section{Remède atikamekw}

http://www.wapikoni.ca/films/remede-atikamekw

Réalisation : Martine Denis-Damée

Année de production : 2012

Durée : 5:32 min.

Communauté : Opitciwan

Ce court documentaire présente la récolte et la préparation d'un remède atikamekw nehirowisiw à partir d'une plante médicinale. Cette plante permet de mieux dormir, de retrouver la santé et une certaine forme physique. Juliette et sa sœur Béatrice, deux aînées d'Opitciwan, en décrivent le processus de cueillette, les bienfaits ainsi que les différentes étapes de préparation. Le territoire est ici envisagé comme une source d'équilibre et de santé faisant partie intégrale d'un système de guérison. 